\title{
REVIEW
}

\section{Choosing patient-tailored hemodynamic monitoring}

\author{
Cornelis Slagt, Rose-Marieke BGE Breukers and AB Johan Groeneveld* \\ This article is one of ten reviews selected from the Yearbook of Intensive Care and Emergency Medicine 2010 (Springer Verlag) and co-published \\ as a series in Critical Care. Other articles in the series can be found online at http://ccforum/series/yearbook. Further information about the \\ Yearbook of Intensive Care and Emergency Medicine is available from http://www.springer.com/series/2855.
}

\begin{abstract}
Introduction
Currently, the number and (worldwide) availability of techniques for hemodynamic monitoring in the critically ill patient is overwhelming, as nicely summarized elsewhere [1-11]. Techniques vary from completely invasive to non-invasive, from intermittent to continuous, and differ in basic principles, methods, parameters, and costs, among others. The older a device, the more literature is available, but the latter may not always help in choosing hemodynamic monitoring tools for departments or for individual patients, i.e. patient-tailored monitoring.

This chapter is not intended to compare one technique to another, which has been done abundantly in the literature, but to provide a conceptual framework to guide therapy of individual patients in various hospital settings by defining the elements that may help to choose among the available techniques, in the absence of a clear evidence-based survival benefit of any hemodynamic monitoring tool [12-16]. First, a brief discussion of what is available and of underlying basic principles seems warranted, since knowledge of possibilities, limitations and pitfalls is required before responsible choices can be made. We will not address tools to monitor the microcirculation.
\end{abstract}

\section{What do we have and what can they do?}

A physical examination remains the cornerstone of assessing patients with hemodynamic compromise, even though signs and symptoms often poorly predict measured hemodynamic variables [13, 17]. Nevertheless, clinical signs and symptoms help to clearly define the clinical problem and its differential diagnosis. As an

*Correspondence: johan.groeneveld@vumc.nl Department of Intensive Care, VUMC, De Boelelaan 1117, 1081 HV Amsterdam, Netherlands adjunct, some type of hemodynamic monitoring is often decided upon, depending on the clinical severity of disease and the (department of) presentation of the patient, among other factors. Table 1 briefly summarizes the currently available equipment for advanced hemodynamic monitoring, beyond that of mean arterial pressure (MAP) and heart rate/rhythm. As indicated, a wide variety of hemodynamic parameters can be monitored by the different techniques, in addition to cardiac output. The parameters pertain to cardiac filling and function and its adequacy related to tissue needs. In addition, pulmonary variables pertaining to edema and gas exchange can be assessed with some devices.

There is a large amount of literature concerning the comparability of techniques and derived parameters, such as (absolute values and changes in) cardiac output and preload indicators $[4-7,18,19]$. However, the manner in which the comparability (or clinically important absence thereof) is judged varies greatly among studies. Uniformly accepted criteria to assess the clinical relevance of comparability of monitoring techniques and parameters are lacking. For instance, comparability of techniques for tracking changes and trends in cardiac output may be more relevant in clinical practice than the degree of agreement of absolute values, provided that 'low' and 'high' values can be separated [19]. Moreover, literature on the practical utility of many of these devices and parameters is scarce, so that negativism regarding their practical value may predominate $[16,20]$. There is, however, some literature to suggest that insertion of a pulmonary artery catheter (PAC) and measuring hemodynamic variables may influence the clinical appraisal of hemodynamics at the bedside and may help or prompt the treating physician to change treatment.

Since its introduction in the 1970s, the PAC has indeed become the reference standard for hemodynamic 
Table 1. What do we have and what can they do?

Equipment
Central venous catheter (many companies)
Pulmonary artery catheter and modifications (some companies)
PiCCO" (Pulsion)
LiDCOplus (LiDCO)
NICO (Novametrix)
Modelflow pulse contour analysis (BMI-TNO)
Nexfin (Bmeye)
Flotrac/Vigileo (Edwards Life Sciences)
Pulse-dye densitometry PDD (Nihon Kohden)
Bioimpedance cardiography (Aesculon, Osypka Medical)
Hemosonic (Arrow)
CardioQ (Deltex Medical)
Ultrasonic cardiac output monitors (Uscom)
Echocardiographs (some companies)
Parameters
Cardiac pressures and volumes
Cardiac output, flow, velocity/time
Dynamic indices
Cardiac anatomy and regional function
Oxygen-related variables
Carbon dioxide-related variables
Vascular diameters
Manfacturs in partions.

Manufacturers in parentheses.

monitoring and measurement of cardiac output [13-15]. A substantial knowledge database has been built up since then, in a variety of institutions, patient populations, and circumstances [16]. However, in the absence of any rigidly proven survival benefit, the catheter has become discredited in critical care medicine [12-16]. The lack of apparent benefit may relate, in part, to adverse effects of insertion, improper use, poor interpretation of hemodynamic data, and inadequate treatment decisions based on the collected variables, or combinations of these factors [20]. Conversely, the value of pulmonary artery pressures, pulmonary artery occlusion pressure (PAOP), mixed venous oxygen saturation $\left(\mathrm{SvO}_{2}\right)$, and right heart volumes, some of the variables that can be uniquely assessed at the bedside of the critically ill patient with help of the PAC and right-sided thermodilution, remains hotly debated $[13-15,20]$. The patient population or circumstance that is most likely to benefit from pulmonary artery catheterization is, therefore, still being actively looked for [13-15, 21, 22].

A second generation hemodynamic monitoring principle includes the less invasive transpulmonary (dye) thermodilution technique, e.g. PiCCO. This technique offers the unique possibility of estimating cardiac preload volumes, measurements of which are not confounded by mechanical ventilation in contrast to pressure and dynamic indices of preload and fluid responsiveness, and of extravascular lung water as a direct measure of pulmonary edema and permeability. Dilutional methods to measure cardiac output include the transpulmonary lithium and indocyanine green (pulse dye) techniques, allowing peripheral injections and peripheral and, for pulse dye, non-invasive detection.

Pulse-contour or pulse-power methods, needing relatively frequent recalibration for optimal performance in tracking changes in cardiac output, are often incorporated in dilutional cardiac output measurement devices needing arterial access $[5,18]$. Some of these methods are truly non-invasive, however. The algorithms used differ from one method to the other, some perform better than others, and the need for recalibration upon changes in time or in vascular tone upon treatment continue to limit their independent applicability $[5,18]$. Calibration can also be performed by ultrasonically obtained aortic diameter for the otherwise well performing Model flow method [23]. The algorithm used in the latter method computes the aortic flow waveform from pulsating arterial blood pressure by simulating a nonlinear, self-adaptive (three-element Windkessel) model of the aortic input impedance. Characteristic impedance and compliance of the aorta non-linearly depend on arterial pressure, and peripheral resistance adapts to changes in blood flow. The degree of non-linearity depends on the subject's sex, age, height, and weight.

An arterial waveform analysis without external calibration, the FloTrac/Vigileo system, is supposed to be relatively independent of vascular tone [9]. Each arterial waveform detected via an arterial catheter is analyzed with a frequency of $100 \mathrm{~Hz}$. The arterial waveform is analyzed for 8 different characteristics, including the upstroke and downslope of the curve. Each curve is analyzed separately and additional curves are analyzed and compared with former and subsequent curves. From this analysis, which takes 20 seconds, the average curve is given, by means of the standard deviation of the given characteristics of the curves. From the given stroke volume and heart rate, the cardiac output is determined, which is updated every 20 seconds. A filter is embedded in the computer to adjust for excesses in systolic blood pressures and heart rates. The accuracy of this method has increased with consecutive software versions.

Doppler ultrasound methods estimate cardiac output by measuring aortic blood flow velocity [10, 11, 24, 25] and multiplying it by the cross-sectional area of the aorta at the insonation point. The probe is introduced orally or nasally and placed at the level of the descending aorta. Some systems measure the descending aortic diameter; others use a monogram to estimate it. Limitations of the 
technique include operator-dependency in finding the optimal angle of insonation, turbulent flow, and changes in relative perfusion of upper and lower body parts via the aorta. Obviously, echocardiography yields clinically useful information on cardiac anatomy and (regional) function that is hard to obtain otherwise, in addition to non-unique parameters, such as cardiac filling and output $[26,27]$. The technique is highly dependent on available expertise and commitment.

\section{Factors affecting choices}

Tables 2-4 describe the issues that may be relevant for decision making, including theoretical considerations, the hardware involved, and patient-bound factors. Indeed, demands put on technologies may vary according to need in different hospital environments and patient populations. We will highlight just some of the considerations mentioned in the Tables. Table 2 essentially notes theoretical considerations, suggesting that the ideal hemodynamic monitoring tool should be simple, safe, relatively versatile, uniformly applicable and beneficial for survival in each patient subjected to that tool, at low or at least affordable costs. Obviously, no method yet fits this 'ideal' list, and perhaps never will, so some compromise on these issues remains necessary.

Some hemodynamic optimization strategies, such as fluid management guided by prediction of fluid responses, early goal-directed therapy, and perioperative hemodynamic optimization or fluid restriction, may help to improve patient outcomes, in terms of reducing complications, lengths of stay, and prevention of overhydration, for example, even irrespective of vital status $[1,16,25,28-33]$. Devices and parameters to assess fluid responsiveness include transpulmonary dilution-derived cardiac volumes, esophageal Doppler flow and echocardiographic indices, and dynamic indices provided by pulse-contour methods $[10,11,24,25,33,34]$. In contrast, central venous pressure (CVP) monitoring may suffice in successful fluid restriction policies [32]. The well-known outcome (survival) benefit of early goaldirected therapy in septic shock, with treatment guided by CVP, central venous oxygen saturation $\left(\mathrm{ScVO}_{2}\right)$ and MAP, has been confirmed by others, since the landmark paper by Rivers et al. [35] and this approach is included in current guidelines on the management of septic shock $[1,31]$, even though CVP may poorly predict fluid responses [36]. Hence, monitoring tools could be judged on their ability to provide parameters that help physicians to implement the strategies mentioned, even if these are slightly different from those originally used in demonstrating benefit but apply similar physiologic and clinical concepts $[1,15,30,37-39]$. For example, the benefit of perioperative hemodynamic optimization with help of the PAC [28], transpulmonary/lithium dilution [29, 30],
Table 2. Theoretical considerations for choosing among hemodynamic monitoring tools

\begin{tabular}{l}
\hline - Safety and side effects \\
- Versatility, number, relevance and utility of parameters \\
- Can be utilized by nurses and physicians: Ease of use, user-friendliness, \\
education, learning curve \\
- Possibilities for assessing fluid responsiveness, goal-directed therapy \\
and other resuscitation strategies of proven outcome benefit even if not \\
decreasing mortality \\
- Demonstrated treatment alterations \\
- Acceptable cost-effectiveness
\end{tabular}

Table 3. Hardware considerations for choosing among hemodynamic monitoring tools

- $\quad$ Availability
- $\quad$ Expertise: Personal, colleagues, and in the literature
- $\quad$ Level of integration in monitors
- $\quad$ Uniformity of applicability
- $\quad$ Continuous vs intermittent
- $\quad$ Invasive vs non-invasive
- $\quad$ Accuracy/reproducibility of parameters
- $\quad$ Response time to interventions and accurate trending

Table 4. Patient-bound considerations for tailoring hemodynamic monitoring
Cardiac rhythm, function, and valvular disease
- Mechanical ventilation:Tidal volume, frequency, positive end-expiratory pressure
- $\quad$ Type, severity and stage of (anticipated) disease warranting hemodynamic monitoring, such as shock and acute lung injury
- Type of circulatory support and change contemplated therein: Fluids, drugs, devices for circulatory support
- Vascular access and other anatomic factors (contraindications)
- Tolerance

esophageal Doppler [10, 11, 24, 25], or dynamic indices [38] could translate into a benefit of optimization of central/mixed venous oxygen saturation since all are intended to optimize tissue oxygenation [37]. Nevertheless, not all devices and parameters have been successfully evaluated yet in hemodynamic optimization strategies and these issues continue to be subject to ongoing research and debate $[1,15,37,39,40]$. Thus, we may need to formulate and test hemodynamic monitoring strategies, rather than to evaluate performance and efficacy of single devices and parameters. The rationale of these strategies may be enforced if led by physiological and clinical considerations as well as by epidemiological and economic issues. Finally, effectiveness could be 
defined in terms of the clinical utility of devices and parameters that may go beyond their formally reported efficacy.

Hardware considerations (Table 3) include the environment where the hemodynamic monitoring is used. Different departments may have different facilities, patient populations and staffing, and pressures on time by emergencies may drive choices for less invasive techniques that can be applied immediately by most of the available staff. Non-invasive hemodynamic monitoring devices may also be of help in departments without facilities for invasive techniques, such as step-down units, long-term facilities, and stroke units. By virtue of definition, any device that is able to accurately detect rapid changes in cardiac output upon fluid challenge would suffice in evaluating fluid responsiveness and some methods may be too slow to fulfill this criterion.

General considerations regarding patient-bound factors (Table 4) include the notion that the sicker the patient the greater the need for accurate hemodynamic parameters to be collected to supplement clinical judgment and the greater likelihood that invasive, rather than less invasive, techniques will meet these needs. In the patient with severe septic shock admitted to the intensive care unit (ICU) for instance, non-invasive arterial waveform analysis-derived cardiac output measurements are less useful as they are affected by vascular tone and require repeated recalibration, at least in the initial resuscitation phase. In patients with or at great risk of pulmonary edema, hemodynamic monitoring by transpulmonary dilution and measurements of extravascular lung water could be chosen to help to prevent harmful overhydration and prolonged mechanical ventilation, unless the patient will anyway need to be intubated and mechanically ventilated. Catheters in the femoral artery are relatively contraindicated during/after aortic-bifemoral reconstruction, and transesophagal echocardiography is not feasible during/after esophageal resection. Esophageal disease may be a contraindication for the use of esophageal Doppler probes, which are also poorly tolerated in awake, non-intubated patients $[10,20,25]$. The presence of cardiac disease and mechanical ventilation may also affect choices. It is likely that a PAC and measurement of PAOP is more helpful in guiding (fluid) management in the presence of systolic/diastolic cardiac dysfunction than during hypovolemic shock, for example [21, 34]. In severe left-sided valvular disease, right-sided measurements of cardiac output are probably preferable to transpulmonary ones, even though the debate on the confounding effect of even minimal tricuspid regurgitation on these measurements has not yet ended. In the presence of endocarditis, intracardiac catheters may be relatively contraindicated. In contrast, a suspected ventricular septal defect may require monitoring with help of a
PAC, echocardiography, or both. In mechanically ventilated patients, filling pressures that are confounded by airway pressures may be less useful in predicting and guiding fluid responses than volumetric preload measurements [34,36], whereas the currently proposed superiority of dynamic indices [33] can be questioned, as they are affected by ventilatory frequency and tidal volume. Finally, pulse-contour methods are sensitive to arrhythmias, aortic valve regurgitation, intra-aortic balloon pumping and peripheral vascular disease.

\section{Conclusions and perspective}

This chapter attempts to provide a conceptual framework for choosing patient-tailored hemodynamic monitoring from available techniques, in an era dominated by lack of proven survival benefits for any hemodynamic monitoring device. Decisions for implementing different hemodynamic monitoring devices may improve when systematically considering the relevant issues, according to a predefined checklist, for example. This approach may help to end debates on the use of hemodynamic monitoring equipment from single perspectives only, but obviously choices may differ from one hospital, unit, patient and physician to another, given the variability in facilities, clinical presentations, and expertise. One tool may supplement another, so that it is advisable to gain expertise in more than one method, particularly in training environments. Health technology assessment institutions and agencies can be of help in advising on these complex issues and emergency and intensive care medicine organizations could benefit from their expertise $[1,12,13,25,41]$. The underlying idea, of course, is that helping physicians to direct therapy using numbers rather than signs and symptoms, and helping the medical community by providing clear clinical guidelines on hemodynamic monitoring strategies will effectively result in health care improvements. Perhaps, we also need a new research agenda on these issues.

\section{Abbreviations}

$C V P=$ central venous pressure, $I C U=$ intensive care unit, $M A P=$ mean arterial pressure, $\mathrm{PAC}=$ pulmonary artery catheter, $\mathrm{PAOP}=$ pulmonary artery occlusion pressure, $\mathrm{ScvO}_{2}=$ central venous oxygen saturation.

\section{Competing interests}

CS has received lecture fees from Edwards Lifesciences. RMBGEB and ABJG declare that they have no competing interests.

Published: 9 March 2010

\section{References}

1. Gunn SR, Fink MP, Wallace B: Equipment review: the success of early goaldirected therapy for septic shock prompts evaluation of current approaches for monitoring the adequacy of resuscitation. Crit Care 2005, 9:349-359.

2. Hofer CK, Ganter MT, Zollinger A: What technique should I use to measure cardiac output? Curr Opin Crit Care 2007, 13:308-317.

3. Jhanji S, Dawson J, Pearse RM: Cardiac output monitoring: basic science and clinical application. Anaesthesia 2008, 63:172-181. 
4. Mathews L, Singh KRK: Cardiac output monitoring. Ann Card Anaesth 2008, 11:56-68

5. Maus TM, Lee DE: Arterial pressure-based cardiac output assessment. $J$ Cardiothorac Vasc Anesth 2008, 22:468-473.

6. Morgan P, Al-Subaie N, Rhodes A: Minimally invasive cardiac output monitoring. Curr Opin Crit Care 2008, 14:322-326.

7. De Waal EEC, Wappler F, Buhre WF: Cardiac output monitoring. Curr Opin Anaesthesiol 2009, 22:71-77.

8. Funk DJ, Moretti EW, Gan TJ: Minimally invasive cardiac output monitoring in the perioperative setting. Anesth Analg 2009, 108:887-897.

9. Mayer J, Boldt J, Poland R, Peterson A, Manecke GR: Continuous arterial pressure wave form-based cardiac output using the Flotrac/Vigileo: a review and meta-analysis. J Cardiothorac Vasc Anesth 2009, 23:401-406.

10. Schober P, Loer SA, Schwarte LA: Perioperative hemodynamic monitoring with transesophageal Doppler technology. Anesth Analg 2009, 109:340-353.

11. Singer M: Oesophageal Doppler. Curr Opin Crit Care 2009, 15:244-248.

12. Scales DC, Sibbald WJ: Medical technology in the intensive care unit. Curr Opin Crit Care 2004, 10:238-245.

13. Harvey S, Stevens K, Harrison D, et al:: An evaluation of the clinical and costeffectiveness of pulmonary artery catheters in patient management in intensive care: a systematic review and a randomized controlled trial. Health Technol Assess 2006, 10:1-152

14. Shah MR, Hasselblad V, Stevenson LW, et al.: Impact of the pulmonary artery catheter in critically ill patients. Meta-analysis of randomized clinnical trials. JAMA 2005, 294:1664-1670.

15. Antonelli M, Levy M, Andrews PJ, et al.: Hemodynamic monitoring in shock and implications for management. International Consensus Conference, Paris, France, 27-28 April 2006. Intensive Care Med 2007, 33:575-590.

16. Ospina-Tascón GA, Cordioli RL, Vincent JL: What type of monitoring has been shown to improve outcomes in acutely ill patients ? Intensive Care Med 2008, 34:800-820

17. Sevransky J: Clinical assessment of hemodynamically unstable patients Curr Opin Crit Care 2009, 15:234-238.

18. De Wilde RB, Schreuder JJ, van den Berg PC, Jansen JR: An evaluation of cardiac output by five arterial pulse contour techniques during cardiac surgery. Anaesthesia 2007, 62:760-768.

19. Squara $P$, Cecconi M, Rhodes $A$, Singer M, Chiche JD: Tracking changes in cardiac output: methodological considerations for the validation of monitoring devices. Intensive Care Med 2009, 35:1801-1808.

20. Vincent JL, Pinsky MR, Sprung $C L$, et al.: The pulmonary artery catheter: in medio virtus. Crit Care Med 2008, 36:3093-3096.

21. Ranucci M: Which cardiac surgical patients can benefit from placement of a pulmonary artery catheter? Crit Care 2006, 10(Suppl 3):S6.

22. Harvey SE, Welch CA, Harrison DA, Rowan KM, Singer M: Post hoc insights from PAC-Man-The UK pulmonary artery catheter trial. Crit Care Med 2008, 36:1714-1721

23. de Vaal JB, de Wilde RB, van den Berg PC, Schreuder JJ, Jansen JR: Less invasive determination of cardiac output from the arterial pressure by aortic diameter-calibrated pulse contour. Br J Anaesth 2005, 95:326-331.

24. Abbas SM, Hill AG: Systematic review of the literature for the use of oesophageal Doppler monitoring for fluid replacement in major abdominal surgery. Anaesthesia 2008, 63:44-51.

25. Mowatt G, Houston G, Hernández R, de Vert: Systematic review of the clinical effectiveness and cost-effectiveness of oesophageal Doppler monitoring in critically ill and high-risk surgical patients. Health Technol Assess 2009, 13:1-120.
26. Slama M, Maizel J: Echocardiographic measurement of ventricular function. Curr Opin Crit Care 2006, 12:241-248.

27. Salem R, Vallee F, Rusca M, Mebazaa A: Hemodynamic monitoring by echocardiography in the ICU: the role of the new echo techniques. Curr Opin Crit Care 2008, 14:561-568

28. Kern JW, Shoemaker WC: Meta-analysis of hemodynamic optimization in high-risk patients. Crit Care Med 2002, 30:1686-1692.

29. Pearse R, Dawson D, Fawcett J, Rhodes A, Grounds RM, Bennett ED: Early goal-directed therapy after major surgery reduces complications and duration of hospital stay. A randomised, controlled trial [ISRCTN38797445]. Crit Care 2005, 9:R687.

30. Goepfert MSG, Reuter DA, Akyol D, Lamm P, Kilger E, Goetz AE: Goal-directed fluid management reduces vasopressor and catecholamine use in cardiac surgery patients. Intensive Care Med 2007, 33:96-103.

31. Rivers EP, Coba V, Whitmill M: Early goal-directed therapy in severe sepsis and septic shock: a contemporary review of the literature. Curr Opin Anesthesiol 2008, 21:128-140.

32. Stewart RM, Park PK, Hunt JP, et al.: Less is more: improved outcomes in surgical patients with conservative fluid administration and central venous catheter monitoring. J Am Coll Surg 2009, 208:725-737.

33. Marik PE, Cavalazzi R, Vasu T, Hirani A: Dynamic changes in arterial waveform derived variables and fluid responsiveness in mechanically ventilated patients: a systematic review of the literature. Crit Care Med 2009, 37:2642-2647.

34. Breukers RM, Trof RJ, de Wilde RB, et al:: Relative value of pressures and volumes in assessing fluid responsiveness after valvular and coronary artery surgery. Eur J Cardiothorac Surg 2009, 35:62-68.

35. Rivers E, Nguyen B, Havstad S, et al:: Early goal-directed therapy in the treatment of severe sepsis and septic shock. N Engl J Med 2001, 345:1368-1377.

36. Marik PE, Baram M, Vahid B: Does central venous pressure predict fluid responsiveness? A systematic review of the literature and the tale of seven mares. Chest 2008, 134:172-178.

37. Collaborative Study Group on Perioperative $\mathrm{S}_{c v} \mathrm{O}_{2}$ : Monitoring Multicentre study on peri- and postoperative central venous oxygen saturation in high-risk surgical patients. Crit Care 2006, 10:R158.

38. Lopes MR, Oliveira MA, Pereira VOC, Lemos IPB, Auler JOC, Michard F: Goal-directed fluid management based on pulse pressure variation monitoring during high-risk surgery: a pilot randomized controlled trial. Crit Care 2007, 11:R100.

39. Buettner M, Schummer W, Hiettemann E, Schenke S, van Hout N, Sakka SG: Influence of systolic-pressure-variation-guided intraoperative fluid management on organ function and oxygen transport. Br J Anaesth 2008, 101:194-199.

40. Uchino S, Bellomo R, Morimatsu H, et al:: Pulmonary artery catheter versus pulse contour analysis: a prospective epidemiological study. Crit Care 2006, 10:R174.

41. Oortwijn WJ, Hanney SR, Ligtvoet A, et al:: Assessing the impact of health technology assessment in the Netherlands. Int J Technol Assess Health Care 2008, 24:259-269.

doi:10.1186/cc8849

Cite this article as: Slagt $C$, et al:: Choosing patient-tailored hemodynamic monitoring. Critical Care 2010, 14:208. 